\title{
Thermal radiation and inclusive production in the running coupling $k_{T}$ - factorization approach
}

\author{
A. V. Giannini ${ }^{1}$, V.P. Goncalves ${ }^{2}$ and P.V.R.G. Silva ${ }^{2}$ \\ 1 Instituto de Física, Universidade de São Paulo, \\ Rua do Matão 1371, 05508-090 São Paulo-SP, Brazil \\ 2 Instituto de Fúsica e Matemática, \\ Universidade Federal de Pelotas \\ Caixa Postal 354, CEP 96010-900, Pelotas, RS, Brazil
}

\begin{abstract}
The characteristics of the thermal radiation are investigated using a two - component model, with the hard component being described by the Color Glass Condensate formalism. The inclusive transverse momentum spectra of charged hadrons produced in proton - proton and proton - nucleus collisions at LHC energies and large $-p_{T}$ are estimated using the running coupling $k_{T}$ - factorization formula and the solution of the Balitsky - Kovchegov equation. Our results indicate that the thermal term is necessary to describe the experimental data and that the effective thermal temperature has an energy dependence similar to the saturation scale. We demonstrate that the enhancement of the thermal temperature in $p P b$ collisions is consistent with that predicted by the saturation scale.
\end{abstract}

PACS numbers:

Keywords: Particle production; QCD dynamics; Thermal radiation; Hadronic collisions

\section{INTRODUCTION}

The description and understanding of the inclusive particle production in hadronic collisions at high energies is still one of the main challenges of the strong interactions theory - the Quantum Chromodynamics (QCD). While the production of particles with a large transverse momentum $p_{T}$, usually denoted hard regime, is quite well described by perturbative QCD in terms of the scattering between quarks and gluons, with their subsequent fragmentation, the main contribution for the transverse momentum spectra comes from particles with small $p_{T}$, which characterizes the soft regime. The mechanism of production of these particles is still not well understood due to the dominance of nonperturbative effects. In addition, the particle production in the semi - hard regime, present between the soft and hard regimes, is expected to be modified by nonlinear QCD effects due to high density of gluons in the initial state. At large energies, the wave function of the projectiles is probed at small Bjorken $x$, being characterized by a large number of gluons. The dense system of partons is predicted to form a new state of matter - the Color Glass Condensate (CGC) - where the gluon distribution saturates and nonlinear coherence phenomena dominate (For a review see e.g. (1]). Such a system is endowed with a new dynamical momentum scale, the saturation scale $Q_{s}$, which controls the main characteristic of the particle production and whose evolution is described by an infinite hierarchy of coupled equations for the correlators of Wilson lines 2-4. As for large energies $Q_{s}$ becomes much larger than the QCD confinement scale $\Lambda_{Q C D}$, the presence of the nonlinear effects is expected to affect the soft particle production.

Recent phenomenological studies [5/7] have demonstrated that the transverse momentum distribution of charged hadrons produced in high-energy hadronic collisions can be accurately described by a two - component model, given by the sum of an exponential (thermal) component and a power - law (pQCD - inspired) term. Despite accounting for the bulk of the produced hadrons, an universal exponential shape for the soft part of the spectra is only partially understood. On one hand, such exponential behavior is expected in heavy-ion collisions $[8]$ as it is usually associated with the thermalization of the produced system, naturally achieved by the continuous redistribution of energy through final-state interactions. On the other hand, its appearance in proton-proton collisions is quite surprising. Given the (much lower) final multiplicity observed in these collisions, a thermal behavior arising through a series of final-state interactions seems quite unlikely. Such result has motivated the proposal of alternative models to describe the origin of the thermal behavior in the transverse momenta spectra. In Ref. 9], by describing the initial wave function of a colliding system according to the CGC formalism, the authors associated the thermal behavior to the emergence of an event horizon resulting from the rapid deceleration of the colliding hadron induced by strong longitudinal color fields, in analogy with the Hawking-Unruh effect. The effective temperature of the thermal contribution was associated with the hard scale of the problem, the saturation scale, $Q_{s}$. In Refs. [6, 7], the thermal contribution has also been associated to the high degree of quantum entanglement in the hadronic wave-function. More recently, the connection between the thermal behavior and the CGC formalism has been discussed in Refs. 10, 11. In particular, such studies have demonstrated that, even changing the model for the hadronization, the transverse momentum spectra cannot be described without a thermal radiation term. 
Our goal in this paper is to improve our understanding about the thermal radiation term, mainly focusing on the energy dependence of the effective temperature. As in Refs. [5-7], we will consider the two - component model, but instead of a parametrization for the description of the hard component, we will estimate the spectra using the CGC formalism taking into account of the nonlinear effects in the QCD dynamics. In particular, we will use in our calculations the running coupling $k_{T}$ - factorization formula derived in Ref. [12] and the solution of the Balitsky Kovchegov equation [2, 3]. As a consequence, we will be able to derive realistic predictions for the spectra in the semi - hard regime. Such approach will also allow us to investigate in more detail the magnitude and energy dependence of the thermal component and the possible relations between the thermal radiation behavior and the CGC formalism.

The paper is organized as follows. In the next Section, we will present a brief review of the formalism, with particular emphasis in the running coupling $k_{T}$ - factorization formula. In Section III the main parameters of the model are determined using the recent LHC data for the transverse momentum spectra of hadrons in $p p$ and $p P b$ collisions. Results for the energy dependence of the effective temperature are presented and the magnitude of the thermal radiation contribution is estimated. Finally, in Section [V] we summarize our main conclusions.

\section{FORMALISM}

During the last decades, a large amount of data on hadron production in proton - proton, proton - nucleus and nucleus - nucleus has been accumulated. The analysis of these data indicate that the momentum distribution of charged particles present distinct behaviors depending on the $p_{T}$ region one is interest. In particular, the data in the soft region $\left(\right.$ low $\left.-p_{T}\right)$, can be described in terms of an exponential function in the transverse mass $m_{T}=\sqrt{m^{2}+p_{T}^{2}}$ ( $m$ is the hadron mass), similar to the Boltzmann spectrum. As pointed out before, the presence of this thermal behavior in collisions of small objects, as $p p$ collisions, is still a theme of intense debate. On the other hand, the experimental data for the inclusive hadron production at large $p_{T}$ can quite well be described using perturbative QCD, with the transverse momentum spectra being expressed in terms of the parton distributions of the incident hadrons, fragmentation functions and partonic cross sections. Such distinct behaviors have motivated the authors from Refs. [5, 6] to propose the description of the invariant yield as a sum of the thermal and hard components, as follows

$$
\frac{d N}{d y d^{2} p_{T}}=F_{\mathrm{th}}\left(p_{T}\right)+F_{\mathrm{hard}}\left(p_{T}\right)
$$

As in Refs. [5] 7, 10, 11] we will parametrize the thermal component by

$$
F_{\mathrm{th}}=A_{\mathrm{th}} \exp \left(-m_{T} / T_{\mathrm{th}}\right)
$$

where the normalization $A_{\text {th }}$ and the effective thermal temperature $T_{\mathrm{th}}$ are free parameters. From perturbative QCD, it is expected that the hard component will have a $1 / p_{T}^{n}$ behavior at large transverse momentum, which have motivated the authors to parametrize this component as follows

$$
F_{\text {hard }}=A_{\text {hard }}\left(1+\frac{m_{T}^{2}}{n T_{\mathrm{h}}^{2}}\right)^{-n}
$$

where $A_{\text {hard }}, T_{\mathrm{h}}$ and $n$ are free parameters to be determined from the fit to the experimental data. Although this parametrization captures the main theoretical expectations and allow us to establish a relation between $T_{\mathrm{th}}$ and $T_{\mathrm{h}}$, we believe that a more quantitative study can be performed if instead of a parametrization for $F_{\text {hard }}$ we estimate this quantity using a formalism based on first principles, as the CGC formalism. Our study is strongly motivated by Refs. 9 11] which demonstrated that the temperature $T_{\mathrm{th}}$ is proportional to the saturation scale $Q_{s}$, establishing a strict connection between the thermal and hard components and sheds a light on the origin of the thermal radiation.

At large energies and central rapidities, the hadronic wave functions of the incident particles are characterized by a large number gluons with small values of the Bjorken - $x$ variable. In this regime, we expect that the QCD dynamics will be described by the CGC effective field theory [1, 13. Due to the large occupation number of gluons at high energies the CGC framework assumes the colliding system can be treated classically, meaning the dynamics of particle production are determined by the Classical Yang-Mills equations [14, 15. For proton-proton and proton-nucleus collisions such equations can be linearized and solved analytically [16 18. By doing so, one obtain an $k_{T}$-factorized expression for the inclusive gluon production cross section whose main inputs are the unintegrated gluon distributions (UGDs) [16 of the incident particles, representing the probability of finding a gluon with momentum fraction $x$ and transverse momentum $k_{T}$ in a hadron $h_{i}$. By being a product of a classical calculation, the expression derived in [16] is valid for fixed coupling. The inclusion of running coupling effects was later considered in [12] by resumming the 
subset of Feynman diagrams related with such effects on top of the leading order expression. The running coupling corrected $k_{T}$-factorization formula reads ${ }^{1}$ :

$$
\frac{d \sigma^{g}}{d y d^{2} k_{T}}=\frac{2 C_{F}}{\pi^{2}} \frac{1}{\boldsymbol{k}^{2}} \int d^{2} q \bar{\phi}_{h_{1}}\left(\boldsymbol{q}, x_{1}\right) \bar{\phi}_{h_{2}}\left(\boldsymbol{k}-\boldsymbol{q}, x_{2}\right) \frac{\alpha_{s}\left(\Lambda_{\mathrm{coll}}^{2} e^{-5 / 3}\right)}{\alpha_{s}\left(Q^{2} e^{-5 / 3}\right) \alpha_{s}\left(Q^{* 2} e^{-5 / 3}\right)},
$$

where $C_{F}=\left(N_{c}^{2}-1\right) / 2 N_{c}, x_{1,2}$ being the momentum fraction of the projectile and the target, respectively, and $\Lambda_{\text {coll }}^{2}$ is a collinear infrared cutoff. Moreover, $\bar{\phi}_{h_{i}}(\boldsymbol{k}, x)$ denotes the UGD for each colliding hadron [12],

$$
\bar{\phi}(\boldsymbol{k}, y)=\frac{C_{F}}{(2 \pi)^{3}} \int d^{2} b d^{2} r e^{-i \boldsymbol{k} \cdot \boldsymbol{r}} \nabla_{r}^{2} \mathcal{N}_{A}(\boldsymbol{r}, y, \boldsymbol{b}),
$$

and do not contain a factor of $1 / \alpha_{s}\left(k^{2}\right)$ as in the fixed coupling case. Such factors now appear explicitly in the denominator of Eq. (4) with the appropriate scale whose expression can be found in [12, 19, 20. The quantity $\mathcal{N}_{A}(\boldsymbol{r}, y, \boldsymbol{b})$ denotes the forward dipole scattering amplitude in the adjoint representation at fixed impact parameter $\boldsymbol{b}$. Following previous works [20 25] an uniform gluon density within the proton has been assumed. As a direct consequence, $\mathcal{N}_{A}(\boldsymbol{r}, y, \boldsymbol{b})=\mathcal{N}_{A}(\boldsymbol{r}, y) S(\boldsymbol{b})$, where $S(\boldsymbol{b})$ is the profile function of the target, and the integration over the impact parameter in Eq. (5) can be performed, generating a factor proportional to the effective interaction area of the colliding hadrons. In our study, $\mathcal{N}_{A}(\boldsymbol{r}, y)$ will be given by the solution of the running coupling Balitsky-Kovchegov (rcBK) equation [26. While results for bulk observables in heavy-ion collisions may not differ much when using UGD sets associated with different initial conditions for the rcBK equation [20, 27, the results obtained with the McLerran-Venugopalan model as initial condition does not provide the best description of the lepton-hadron data at HERA energies [28] nor the $p_{T}$-spectra in proton-proton collisions [25]. Therefore, in our study we will estimate the gluon spectrum using the "g1.101" UGD set from Ref. [25].

In order to estimate the single inclusive hadron distribution, we need to convolute Eq. (4) with the fragmentation function for gluons into charged hadrons

$$
F_{\text {hard }}\left(p_{T}\right)=\frac{d N^{h}}{d y d^{2} p_{T}}=\frac{K}{\sigma_{\text {inel }}} \int \frac{d z}{z^{2}} \frac{d \sigma^{g}}{d y d^{2} k_{T}} \times D_{g}^{h}\left(z=\frac{p_{T}}{k_{T}}, \mu^{2}\right),
$$

where $\sigma_{\text {inel }}$ is the inelastic cross section and the $K$-factor mimics the effect of higher order corrections and, effectively, of other dynamical effects not included in the CGC formulation. Such factor is treated as a free parameter, which is a recurrent assumption in phenomenological studies. In our calculations, we will make use of the KKP fragmentation functions [29]. It is worth noting that the convolution also fix the collinear infrared cutoff $\Lambda_{\text {coll }}^{2}$ as it should match the momentum scale $\mu^{2}$ used in the fragmentation model [30. The above expression is strictly valid in the perturbative regime of large transverse momentum, where the modelling of the hadronization by a fragmentation function can be applied. In particular, the KKP parametrization, as many others fragmentation sets, has its application limited to semi-hard momenta, usually $p_{T} \geq 1 \mathrm{GeV}$. This limits the application of Eq. (6) to this same range. In order to extend the CGC predictions for softer momenta, we will assume that for $p_{T}<p_{T 0}=1 \mathrm{GeV}$, the inclusive hadron distribution can be modelled by a function $A \exp \left(-m_{T}^{2} / Q_{0}^{2}\right)$, where $A$ and $Q_{0}$ are fixed by imposing the continuity of the function and of its first derivative. This ensures the hard component has a continuum behavior with $p_{T}$ and that it becomes a constant for $p_{T}=0$. While the extension of the hard component to the soft sector is, of course, model dependent, an exponential behavior is in line with the qualitative expectation that the momentum distribution eventually flats out for $p_{T} \rightarrow 0$ due to the nonlinear QCD effects [14, 31, 33].

\section{RESULTS}

In our analysis of the inclusive hadron production in $p p$ collisions, we will consider data from different experiments of the LHC in the energy range of $0.9 \mathrm{TeV}$ to $13 \mathrm{TeV}$. Namely, data by ALICE Collaboration at energies of $0.9 \mathrm{TeV}$ [34], $2.76 \mathrm{TeV}$ [34], $5.02 \mathrm{TeV}$ [35], $7 \mathrm{TeV}$ [34] and $13 \mathrm{TeV}$ [36]; by ATLAS Collaboration at $7 \mathrm{TeV}$ [37, $8 \mathrm{TeV}$ [38] and $13 \mathrm{TeV}$ [39] and by CMS Collaboration at $0.9 \mathrm{TeV}$ [40, $2.36 \mathrm{TeV}$ [40] and $7 \mathrm{TeV}$ [41]. We included in the fit all data up to $p_{T}^{\max }=10 \mathrm{GeV}$, in order to optimize the value of $\chi^{2}$. Statistic and systematic errors were added in quadrature.

\footnotetext{
${ }^{1}$ The notation follows the one from ref [12]: $\boldsymbol{k}$ denotes the transverse momentum of the produced gluon while $\boldsymbol{q}$ and $\boldsymbol{k}-\boldsymbol{q}$ are the "intrinsic" transverse momenta from the gluon distributions.
} 

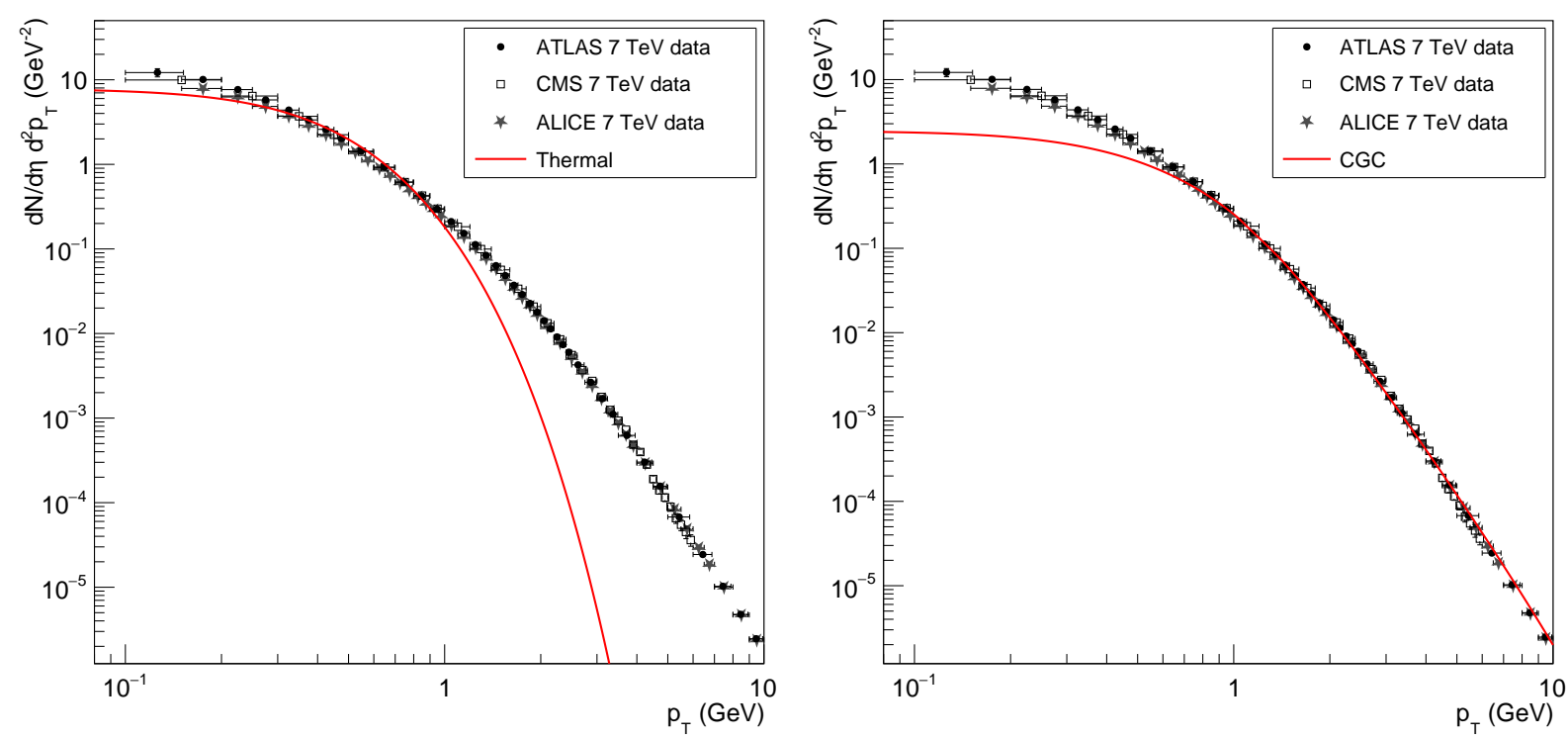

Figure 1: Results for the inclusive transverse momentum spectra of charged hadrons produced in proton-proton collisions at LHC energy considering only either (left panel) the Thermal or (right panel) the CGC component. Data from ALICE 34, ATLAS [37] and CMS [41] Collaborations at $7 \mathrm{TeV}$.

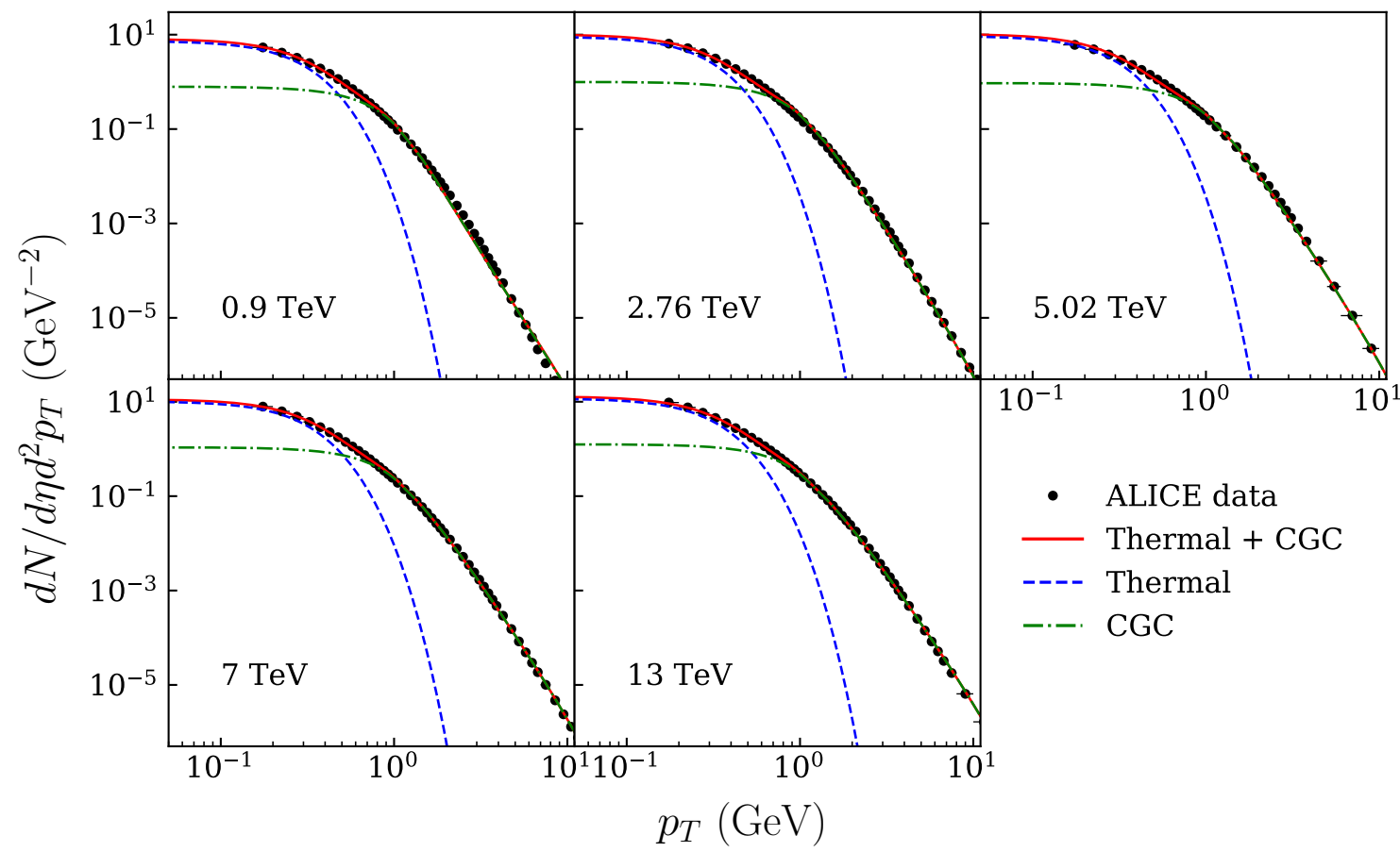

Figure 2: Results for the inclusive transverse momentum spectra of charged hadrons produced in proton-proton collisions for distinct LHC energies considering the Thermal and CGC components. Data from ALICE Collaboration 34, 34, 34, 36].

Moreover, as experimental data at LHC energies are usually presented in terms of pseudorapidity $\eta$ instead of rapidity $y$, the transformation $y \rightarrow \eta$ in Eq. (6) is accounted for by using the effective Jacobian [25]

$$
J(\eta, s)=\frac{\cosh \eta}{\sqrt{\cosh ^{2} \eta+[m / P(s)]^{2}}},
$$




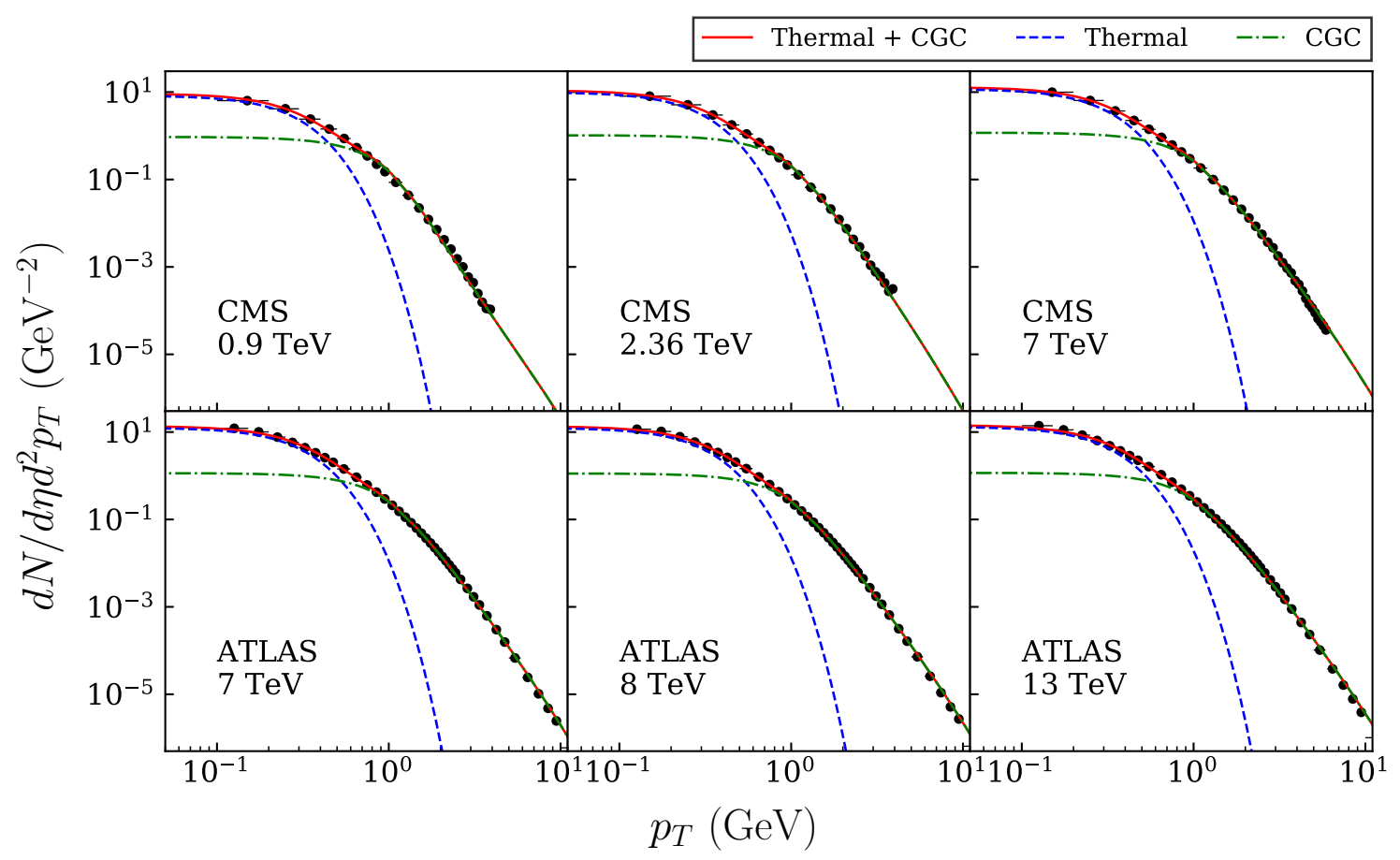

Figure 3: Results for the inclusive transverse momentum spectra of charged hadrons produced in proton-proton collisions for distinct LHC energies considering the Thermal and CGC components. Data from ATLAS [37.39] and CMS 40, 40, 41] Collaborations.

with $P(s)=0.13+0.32\left(\sqrt{s / s_{0}}\right)^{0.115} \mathrm{GeV}$ and an effective mass of $350 \mathrm{MeV}$.

Initially, let's investigate the possibility of describe the experimental data for $p p$ collisions at $\sqrt{s}=7 \mathrm{TeV}$ considering only one of the terms present in Eq. (1). The results of the fit are presented in Fig. 1 considering either only the soft (Thermal) or the hard (CGC) component. The results indicate that both models are not able to describe the data in the full $p_{T}$ range considered. In Figs. 2 and 3 we present our results for the fits of the ALICE and ATLAS/CMS data, respectively, considering the Thermal and CGC contributions. For completeness, the contribution of each component is also shown. As expected, the CGC (Thermal) prediction dominates at large (small) tranverse momentum. It is important to emphasize that the CGC prediction has only one free parameter, the K-factor, which is energy dependent in agreement with previous studies (See e.g. Refs. [25, 42]). A more detailed description of the fits is presented in Table I with some statistical information (reduced $\chi^{2}$ and degrees of freedom, $\nu$ ). The parameters without uncertainty are fixed parameters. Such results demonstrate that the two - component model, Thermal + CGC, describe quite well the current experimental data.

The results for the fits allow us to estimate the energy dependence of the effective thermal temperature $T_{\mathrm{th}}$. Assuming that this quantity can be expressed by

$$
T_{\mathrm{th}}(s)=T_{0}\left[\sqrt{\frac{s}{s_{0}}}\right]^{n}
$$

with $s_{0}=1 \mathrm{GeV}^{2}$ fixed, we have determined $T_{0}=(5.2 \pm 1.3) \times 10^{-2} \mathrm{GeV}$ and $n=0.076 \pm 0.029$, with $\chi^{2} / \nu=0.566$ for $\nu=9$. One has that the effective temperature increases with the energy and that the value of $n$ is compatible with those derived in Refs. 5, 10, 11. In particular, it has a similar energy dependence that the saturation scale, in agreement with the theoretical expectation predicted in Ref. 9] and recently confirmed in Refs. [10, 11]. In order to investigate the impact of the thermal term for different energies, we have estimated the transverse momentum $p_{T}^{*}$, where the thermal and CGC contributions are equal. The results are presented in the left panel of Fig. 4 . One has that $p_{T}^{*}$ slightly increases with the energy, which indicates that the thermal contribution becomes more important at larger energies. We have verified that for ultrahigh cosmic rays energies, $p_{T}^{*}$ is of the order of $0.61 \mathrm{GeV}$. This result motivates a more detailed analysis of the treatment of hadron production in cosmic rays interactions and possible implications of the thermal radiation for the development of air showers.

During the last years, the ALICE Collaboration has released data for the inclusive transverse momentum spectra 


\begin{tabular}{c||c|c||c||c||c}
\hline \hline \multicolumn{1}{c||}{ Energy } & \multicolumn{2}{c||}{$0.9 \mathrm{TeV}$} & $2.36 \mathrm{TeV}$ & $2.76 \mathrm{TeV}$ & $5.02 \mathrm{TeV}$ \\
\hline Experiment & ALICE & CMS & CMS & ALICE & ALICE \\
\hline$A_{\mathrm{th}}$ & $402(134)$ & $587(133)$ & $485(104)$ & $518(182)$ & $550(183)$ \\
$T_{\mathrm{th}}$ & $0.0930(59)$ & $0.0871(38)$ & $0.0951(41)$ & $0.0913(62)$ & $0.0902(51)$ \\
$K$ & $6.27(16)$ & $7.47(15)$ & $4.54(11)$ & $4.040(99)$ & $2.754(58)$ \\
$A$ & 1.26 & 1.50 & 1.56 & 1.50 & 1.39 \\
$Q_{0}^{2}$ & 0.551 & 0.551 & 0.608 & 0.616 & 0.646 \\
\hline$\chi^{2} / \nu$ & 10.74 & 15.16 & 3.44 & 2.10 & 4.86 \\
$\nu$ & 43 & 21 & 21 & 43 & 32 \\
\hline \hline
\end{tabular}

\begin{tabular}{c||c|c|c||c||c|c}
\hline \multicolumn{1}{c||}{ Energy } & \multicolumn{3}{c||}{$7 \mathrm{TeV}$} & \multicolumn{1}{c||}{ TeV } & \multicolumn{2}{c}{$13 \mathrm{TeV}$} \\
\hline Experiment & ALICE & ATLAS & CMS & ATLAS & ALICE & ATLAS \\
\hline$A_{\text {th }}$ & $389(126)$ & $476(89)$ & $434(110)$ & $435(47)$ & $358(70)$ & $393(51)$ \\
$T_{\text {th }}$ & $0.1012(67)$ & $0.1013(37)$ & $0.1021(55)$ & $0.1036(22)$ & $0.1075(45)$ & $0.1085(30)$ \\
$K$ & $2.659(68)$ & $2.822(33)$ & $2.903(71)$ & $2.608(18)$ & $2.284(52)$ & $2.125(19)$ \\
$A$ & 1.56 & 1.66 & 1.71 & 1.63 & 1.77 & 1.65 \\
$Q_{0}^{2}$ & 0.661 & 0.661 & 0.661 & 0.667 & 0.687 & 0.687 \\
\hline$\chi^{2} / \nu$ & 0.739 & 4.14 & 1.99 & 9.49 & 0.992 & 17.2 \\
$\nu$ & 43 & 37 & 31 & 37 & 42 & 38 \\
\hline \hline
\end{tabular}

Table I: Results of fits to experimental data together with statistical information: reduced $\chi^{2}$ and degrees of freedom, $\nu$. Parameters without uncertainty were fixed in the fits. In all fits we considered $1 \sigma$ of confidence level. Parameter $A_{\text {th }}$ and $A$ are given in $\mathrm{GeV}^{-2}, T_{\mathrm{th}}$ and $T$ in $\mathrm{GeV}$ while $K$ is dimensionless.

of charged hadrons produced in $p P b$ collisions 35. We have estimated the CGC contribution and applied the two - component model discussed above for these collisions. The results are presented in the right panel of Fig. 4 by the red dashed line. The associated parameters are the following: $A_{\mathrm{th}}=950(313) \mathrm{GeV}^{-1}, T_{\mathrm{th}}=0.1053(64) \mathrm{GeV}$, $K=3.083(46), A=5.03 \mathrm{GeV}^{-1}, Q_{0}^{2}=0.753 \mathrm{GeV}^{2}$, with $\chi^{2} / \nu=5.46$ for $\nu=43$. We have that the model describes the data. Some interesting conclusions can be obtained by the comparison with the $p p$ results. First, the value of $p_{T}^{*}$ is slightly larger in $p P b$ than in $p p$ collisions, as shown in the left panel of Fig. 4. Such result indicates that the contribution of the thermal term is larger in nuclear collisions, in agreement with the results obtained in Refs. [7, 11. Second, Ref. 9] has derived that $T_{\mathrm{th}} \sim Q_{s}$. As the nuclear saturation scale is expected to enhanced by a factor $A^{1 / 3}$ in comparison to the proton one, i.e. $Q_{s A}^{2} \sim A^{1 / 3} Q_{s p}^{2}$, we expect that $T_{\mathrm{th}}^{p P b} \sim A^{1 / 6} T_{\mathrm{th}}^{p p}$. In the right panel of Fig. 4 we have tested this expectation, by rescaling the effective thermal temperature obtained for $p p$ collisions at $\sqrt{s}=5.02$ $\mathrm{TeV}$ by the factor $1 / 2 A^{1 / 6}$ and estimating the thermal radiation term for this rescaled temperature. The resulting prediction is represented by the blue solid line. One has that the rescaled prediction provides a quite well description of the data, which reinforces the conclusion that the thermal behavior is strictly associated to the treatment of the QCD dynamics at high parton densities, as given by the CGC formalism.

\section{CONCLUSIONS}

One of the more intriguing aspects of the description of inclusive particle production in hadronic collisions at high energies is the presence of a thermal radiation behavior at small transverse momentum. For $p p$ collisions, the impact of final state interactions is expected to be small since such behavior, usually associated to the thermalization of the produced system, is not predicted to be present. Such result have motivated several authors to investigate the connection between the thermal behavior and the high partonic density present in the initial state. At large densities, the nonlinear QCD effects are expected to modify the particle production and to imply the emergence of the thermal spectrum characterized by a temperature proportional to the saturation scale. In this paper we have investigated the characteristics of the thermal radiation term assuming that the hard component of the two - component model is described by the running coupling $k_{T}$-factorization formula, calculated using the solution of the Balitsky - Kovchegov equation. Our analysis improve previous studies that have assumed a parametrization for the hard component. We have demonstrated that the thermal component is needed to describe the inclusive transverse momentum spectra of charged hadrons produced in $p p$ and $p P b$ collisions at the LHC energies. In particular, we shown that this component 


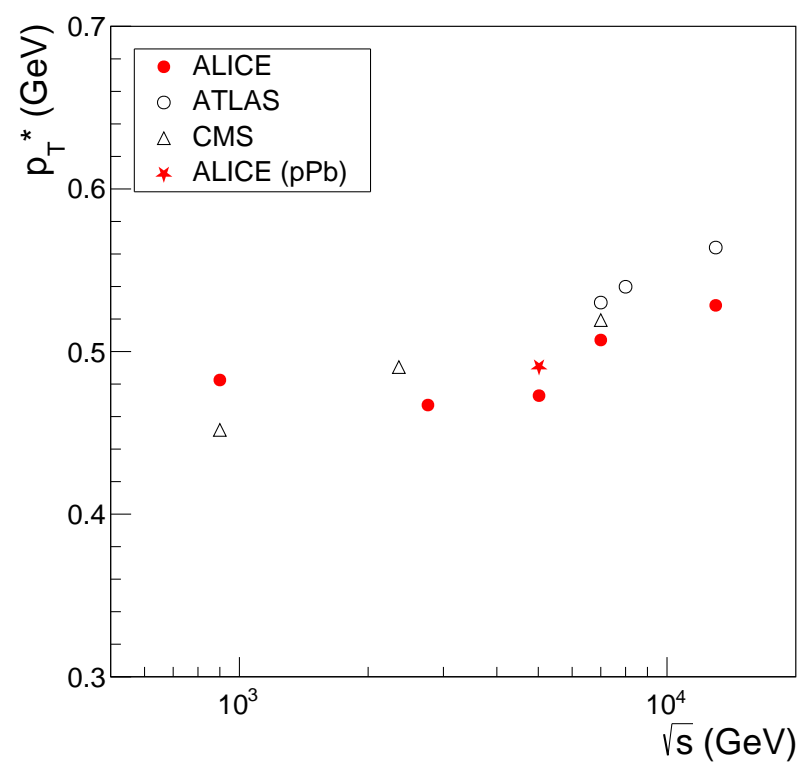

(a)

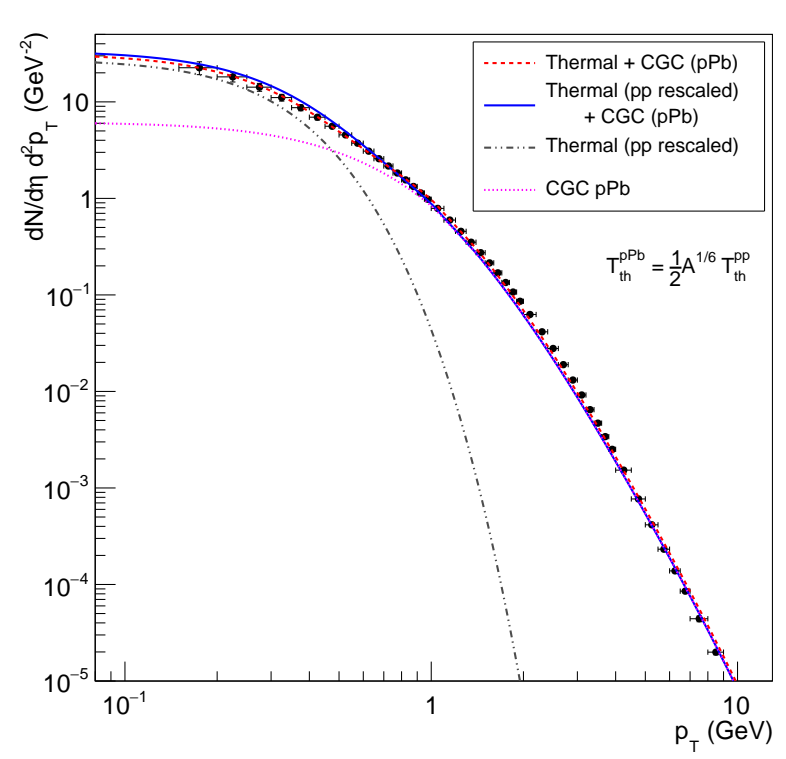

(b)

Figure 4: (a) Energy dependence of the transverse momentum $p_{T}^{*}$, where the thermal and the CGC components are equal. (b) Results for the inclusive transverse momentum spectra of charged hadrons produced in $p P b$ collisions at $\sqrt{s}=5.02 \mathrm{TeV}$. Data from ALICE Collaboration [35.

becomes more important in nuclear collisions and that the nuclear dependence of the effective thermal temperature is compatible with that expected from the relation with the saturation scale.

\section{Acknowledgments}

This work was partially financed by the Brazilian funding agencies CNPq, FAPERGS and INCT-FNA (process number 464898/2014-5 and 155628/2018-6). A.V.G. acknowledges the Brazilian funding agency FAPESP for financial support through grants 2017/14974-8 and 2018/23677-0.

[1] E. Iancu and R. Venugopalan, In *Hwa, R.C. (ed.) et al.: Quark gluon plasma* 249-3363, hep-ph/0303204. H. Weigert, Prog. Part. Nucl. Phys. 55, 461 (2005). F. Gelis, E. Iancu, J. Jalilian-Marian and R. Venugopalan, Ann. Rev. Nucl. Part. Sci. 60, 463 (2010);

[2] I. I. Balitsky, Nucl. Phys. B463, 99 (1996); Phys. Rev. Lett. 81, 2024 (1998); Phys. Rev. D 60, 014020 (1999); Phys. Lett. B 518, 235 (2001).

[3] Y.V. Kovchegov, Phys. Rev. D 60, 034008 (1999); Phys. Rev. D 61074018 (2000).

[4] J. Jalilian-Marian, A. Kovner, L. McLerran and H. Weigert, Phys. Rev. D 55, 5414 (1997); J. Jalilian-Marian, A. Kovner and H. Weigert, Phys. Rev. D 59, 014014 (1999); Phys. Rev. D59, 014015 (1999); Phys. Rev. D 59, 034007 (1999); E. Iancu, A. Leonidov and L. McLerran, Nucl.Phys. A692, 583 (2001); E. Ferreiro, E. Iancu, A. Leonidov and L. McLerran, Nucl. Phys. A701, 489 (2002); H. Weigert, Nucl. Phys. A703, 823 (2002).

[5] A. A. Bylinkin, D. E. Kharzeev and A. A. Rostovtsev, Int. J. Mod. Phys. E 23, no. 12, 1450083 (2014)

[6] O. K. Baker and D. E. Kharzeev, Phys. Rev. D 98, no. 5, 054007 (2018)

[7] X. Feal, C. Pajares and R. A. Vazquez, Phys. Rev. C 99, no. 1, 015205 (2019)

[8] P. F. Kolb and U. W. Heinz, arXiv:nucl-th/0305084 [nucl-th]].

[9] D. Kharzeev and K. Tuchin, Nucl. Phys. A 753, 316-334 (2005)

[10] E. Gotsman and E. Levin, Eur. Phys. J. C 79, no. 5, 415 (2019)

[11] E. Gotsman and E. Levin, Phys. Rev. D 100, no.3, 034013 (2019)

[12] W. A. Horowitz and Y. V. Kovchegov, Nucl. Phys. A 849, 72 (2011).

[13] L. D. McLerran and R. Venugopalan, Phys. Rev. D 49, 2233 (1994); L. D. McLerran and R. Venugopalan, Phys. Rev. D 49, 3352 (1994); L. D. McLerran and R. Venugopalan, Phys. Rev. D 50, 2225 (1994);

[14] A. Kovner, L. D. McLerran and H. Weigert, Phys. Rev. D 52, 6231 (1995) 
[15] A. Kovner, L. D. McLerran and H. Weigert, Phys. Rev. D 52, 3809 (1995)

[16] Y. V. Kovchegov and K. Tuchin, Phys. Rev. D 65, 074026 (2002).

[17] A. Dumitru and L. D. McLerran, Nucl. Phys. A 700, 492 (2002)

[18] J. P. Blaizot, F. Gelis and R. Venugopalan, Nucl. Phys. A 743, 13 (2004)

[19] F. Duraes, A. Giannini, V. P. Goncalves and F. Navarra, Phys. Rev. D 94, no.5, 054023 (2016)

[20] A. Dumitru, A. V. Giannini, M. Luzum and Y. Nara, Phys. Lett. B 784, 417 (2018).

[21] E. Levin and A. H. Rezaeian, Phys. Rev. D 82, 014022 (2010)

[22] E. Levin and A. H. Rezaeian, Phys. Rev. D 82, 054003 (2010)

[23] E. Levin and A. H. Rezaeian, Phys. Rev. D 83, 114001 (2011)

[24] A. Dumitru, D. E. Kharzeev, E. M. Levin and Y. Nara, Phys. Rev. C 85, 044920 (2012)

[25] J. L. Albacete, A. Dumitru, H. Fujii and Y. Nara, Nucl. Phys. A 897, 1 (2013).

[26] Y. Kovchegov, H. Weigert, Nucl. Phys. A 784, 188 (2007)

I. I. Balitsky, Phys. Rev. D 75, 014001 (2007)

E. Gardi, J. Kuokkanen, K. Rummukainen, H. Weigert, Nucl. Phys. A 784, 282 (2007)

I. Balitsky, G. A. Chirilli, Phys. Rev. D 77, 014019 (2008)

[27] A. Dumitru, A. V. Giannini, M. Luzum and Y. Nara, Acta Phys. Polon. Supp. 12, no.4, 973-978 (2019)

[28] J. L. Albacete, N. Armesto, J. G. Milhano and C. A. Salgado, Phys. Rev. D 80, 034031 (2009).

[29] B. A. Kniehl, G. Kramer and B. Potter, Nucl. Phys. B 582, 514 (2000)

[30] Y. V. Kovchegov and H. Weigert, Nucl. Phys. A 807, 158 (2008).

[31] A. Krasnitz, Y. Nara and R. Venugopalan, Phys. Rev. Lett. 87, 192302 (2001)

[32] A. Krasnitz, Y. Nara and R. Venugopalan, Nucl. Phys. A 717, 268 (2003)

[33] D. Kharzeev, E. Levin and K. Tuchin, Phys. Rev. C 75, 044903 (2007)

[34] B. B. Abelev et al. [ALICE Collaboration], Eur. Phys. J. C 73 (2013) no.12, 2662

[35] S. Acharya et al. [ALICE Collaboration], JHEP 1811, 013 (2018)

[36] J. Adam et al. [ALICE Collaboration], Phys. Lett. B 753 (2016) 319

[37] G. Aad et al. [ATLAS Collaboration], New J. Phys. 13 (2011) 053033

[38] G. Aad et al. [ATLAS Collaboration], Eur. Phys. J. C 76 (2016) no.7, 403

[39] M. Aaboud et al. [ATLAS Collaboration], Eur. Phys. J. C 76 (2016) no.9, 502

[40] V. Khachatryan et al. [CMS Collaboration], JHEP 1002 (2010) 041

[41] V. Khachatryan et al. [CMS Collaboration], Phys. Rev. Lett. 105 (2010) 022002

[42] F. Duraes, A. Giannini, V.P. Goncalves and F. Navarra, Phys. Rev. C 94, no.2, 024917 (2016) 